\title{
Estudo Comparativo da Espectrometria de Emissão Atômica com Fonte de Plasma Indutivamente Acoplado com a Espectrometria de Emissão Atômica com Fonte de Centelha para Análise Quantitativa de Aço
}

\author{
Alessandro D. Oliveira ${ }^{1}$, Almir F. Clain ${ }^{2}$ \\ ${ }^{1}$ Universidade Severino Sombra CECETEN, Química, \\ alessandrodioleyahoo.com.br
}

${ }^{2}$ Universidade Severino Sombra, CECETEN, Química, almirclain@hotmail.com

\begin{abstract}
Resumo. Este trabalho é um estudo comparativo de análise de aço de baixa liga empregando duas técnicas de mesmo fundamento básico (excitação de elétrons e emissão de fótons característicos), mas que utilizam fontes de excitação diferentes. As técnicas empregadas foram a espectrometria de emissão atômica com fonte de plasma indutivamente acoplado (ICP-AES) e a técnica de espectrometria de emissão ótica com fonte de centelha (SparkAES).Um estudo estatístico comparou a precisão e a exatidão das técnicas. Os outros parâmetros utilizados na comparação das técnicas foram: a geometria da amostra (sua forma), tipo de amostra, tempos de preparação e análise e fator de recuperação.
\end{abstract}

Palavras-chave: Emissão Atômica com Fonte de Plasma Indutivamente Acoplado, Fonte de Centelha para Análise Quantitativa de Aço.

\section{Introdução}

\subsection{O Aço}

A maior parte do ferro produzido na indústria é convertido em alguma forma de aço. Podem-se adicionar outros metais (p.ex. W, Cr, Mo, V, Mn, Ni, Co) em quantidades consideráveis ou não, para produção de ligas de aço destinadas a fins especiais. [MELLOR, 1955, p.967]. Além disso, há outros elementos decorrentes do processo de fabricação, tais como P, S, Mn, Si, Sn, Al, N, O e H. A análise química do aço é um dos ensaios de controle para o processo de produção. [SILVA, 1988, p.421].

Para se produzir uma liga de aço, um forno é abastecido com gusa ou sucata. Após a fusão e homogeneização uma amostra é coletada e enviada ao laboratório químico. O laboratório realiza uma análise completa dos elementos contidos na especificação. $\mathrm{O}$ operador de produção não pode seguir com o processo sem o resultado do laboratório, pois é com o resultado que será cálculo a quantidade de liga que será adicionada para alcançar a liga de aço desejada na especificação. É óbvia a necessidade de uma análise rápida, por dois motivos: devido ao tempo de produção e da representação das condições do banho. [VANCE, 1952, p.114, tradução nossa]

A composição química de um determinado aço é de grande importância, pois é o que define o tipo de aço e sua aplicação. Cada elemento químico define a estrutura e a propriedade do aço. Os efeitos dos elementos de liga nos aços e ligas especiais 
envolvem não apenas alterações nas fases ou constituintes presentes em equilíbrio, mas também na maneira e velocidade com que estas fases se formam. A tabela 1 apresenta alguns elementos e suas principais funções [SILVA, 1988, p.191].

Tabela 1: Principais funções de alguns elementos no aço.

\begin{tabular}{|c|l|}
\hline Elemento & Principais Funções \\
\hline $\mathrm{Al}$ & 1 - Desoxidante eficiente \\
& $2-$ Restringe o crescimento de grão \\
\hline $\mathrm{Cr}$ & 1 - Aumento a resistência à corrosão e à oxidação \\
& 2 - Aumento a endurecibilidade \\
& 3 - Melhora a resistência a altas temperaturas \\
\hline $\mathrm{Mn}$ & 1 - Contrabalança a fragilidade devida ao $\mathrm{S}$ \\
& $2-$ Aumenta a endurecibilidade economicamente \\
\hline $\mathrm{Si}$ & 1 - Desoxidante \\
& $2-$ Elemento de liga para chapas elétricas e magnéticas \\
& $3-$ Aumenta a resistência à oxidação \\
\hline $\mathrm{Mo}$ & $1-$ Eleva a dureza a quente, a resistência a quente e a fluência. \\
& $2-$ Melhora a resistência à corrosão dos aços inoxidáveis \\
& $3-$ Forma partículas resistentes à abrasão. \\
\hline $\mathrm{Ni}$ & 1 - Aumento a resistência de aços recozidos \\
\hline $\mathrm{P}$ & 1 - Aumenta a resistência de aços de baixo C \\
& $2-$ Aumenta a resistência à corrosão \\
& 3 - Aumenta a usinabilidade em aços \\
\hline
\end{tabular}

[SILVA, 1988, p.245-247]

\subsection{Análise de aço por Espectrometria de Emissão Atômica}

A espectrometria aplicada para análise de aço surgiu da necessidade de acelerar o processo de análise, onde até então se utilizavam métodos de via úmida, que eram demorados e de alto custo. Alguns elementos poderiam ser determinados em meia hora, tais como Mn, Cr, Ni, Mo, Cu e V, e outros de 8 a 12h, como Si, W, Al, Zr, Ti, B e Co. [VANCE, 1952, p.115].

A composição química de um aço pode ser determinada por diversas técnicas de análise química, respeitando suas particularidades, mas este trabalho abordará apenas a técnica de espectrometria de emissão por plasma e por centelha.

A técnica de espectrometria por plasma aplicada no estudo, analisa amostras no estado líquido. Amostras sólidas, como o aço (peça maciça, chapa, limalha, cavaco, pequenos pedaços, particulado ou pó) precisam ser convertidas em líquidos por um processo de dissolução.

\section{Revisão da Literatura}

A espectrometria de emissão atômica por centelha começou a ser estudada a partir de 1901e iniciou aplicação generalizada para análise elementar no início da década de 30 . A espectrometria de emissão atômica com fonte de plasma teve grande desenvolvimento na década de 70. [SKOOG, 2004, p.839, tradução nossa].

A técnica de espectrometria, antes de ser utilizada em produção de aço, já era utilizada em outros campos. Desde 1935 muitos equipamentos apareceram neste setor. Este equipamento era chamado de espectrógrafo porque registrava o espectro numa chapa fotográfica. Um espectrógrafo Baird foi instalado em um laboratório de controle em 1943. O equipamento possuía 3 metros de campo focal e rede de difração de 15.000 
linhas por polegada. A fonte de excitação era a centelha proveniente de uma fonte de alta voltagem e chapas fotográficas registravam as linhas espectrais dos fótons característicos emitidos pelos elementos das amostras. A chapa era revelada e a densidade ótica das linhas eram medidas por um densítômetro, e então pelo enegrecimento da linha e construção de uma curva de calibração com padrões, se calculava a concentração dos elementos.

Com o desenvolvimento do espectrômetro de leitura direta, onde sensores de fótons substituíram as chapas fotográficas, pode-se alcançar um tempo de análise de 35 segundos após a amostra ser colocada no equipamento. Este instrumento possuía o mesmo princípio, exceto pela etapa de detecção fotográfica, que foi substituída por sensíveis tubos denominados foto-multiplicadores, que recebiam a luz emitida em vários comprimentos de onda e as enviava para determinados canais já especificados de comprimentos de onda. Em 1947 um espectrômetro também Baird foi instalado num laboratório de controle, sendo o primeiro deste tipo a ser utilizado na indústria de aço. $\mathrm{O}$ equipamento operava $24 \mathrm{~h}$ por dia, sete dias da semana com um operador em cada turno de 8 horas, ao invés de três operadores como com o espectrógrafo. [VANCE, 1952, p.115 e 116].

Cristiane Franchi Petry (2005) avaliou a técnica de ICP-OES para análise de traços de elementos em amostras ambientais, onde determinou as concentrações de As, $\mathrm{Ba}, \mathrm{Cd}, \mathrm{Co}, \mathrm{Cr}, \mathrm{Cu}, \mathrm{Mn}, \mathrm{Ni}, \mathrm{P}, \mathrm{V}$ e $\mathrm{Zn}$ (na ordem de $0,07 \mu \mathrm{g} / \mathrm{L}$ e $0,03 \mu \mathrm{g} / \mathrm{g}$ ).

François R. Doucet, Thomas F. Belliveau, Jean-Luc Fortier e Joseph Hubert (2004) realizaram um estudo comparativo das técnicas de espectrometria por fonte de centelha e espectrometria de plasma induzido por laser (LIPS), para análises de ligas de alumínio, ambos com as mesmas condições óticas - utilizaram as mesmas linhas espectrais. Os resultados obtidos demonstraram que o método LIPS é um método de potencial para análise quantitativa de ligas de alumínio na indústria.

Lílian C. Trevizan, Telma B. Matias e Joaquim A. Nóbrega (2007) avaliaram a viabilidade de análise de aço inoxidável por ICP-OES com vista axial utilizando diversas estratégias de calibração. Conclui-se que a condição robusta do plasma possibilitou a análise de aço inoxidável em ICP-OES com configuração axial utilizando solução padrão de referência em mesmo meio ácido, porém sem os elementos de maior concentração, não ocorrendo severos efeitos de matriz.

\section{Fundamentos da Espectrometria de Emissão Atômica}

\subsection{Emissão de Fótons Característicos}

Quando se introduz um metal em forma de sal em uma chama de um bico de Bunsen, aparecem cores características. Este procedimento é usado há muito tempo para identificar qualitativamente alguns elementos. Se a luz de uma destas chamas passar por um meio de dispersão adequado surgem diversas linhas (ou raias), cada qual com uma cor característica. A cada radiação corresponde um comprimento de onda definido, que lhe atribui uma posição fixa no espectro. [JEFFERY, 1992, p.612].

As determinações espectrométricas de espécies atômicas só podem ser realizadas quando o átomo individual ou íon elementar (como $\mathrm{Fe}^{2+}, \mathrm{Mg}^{+}$ou $\mathrm{Al}^{+}$) estiverem num meio gasoso produzido por uma fonte de energia externa. Essas espécies em níveis de energia excitados produzem fótons com comprimentos de onda suficientemente 
afastados um dos outros para serem detectados por um sensor específico. A primeira etapa do processo de análise é a atomização, na qual uma amostra é volatilizada e decomposta de tal forma que produza íons e átomos em fase gasosa. [SKOOG, 2004, p.839]

O espectro ótico de emissão atômica é composto por uma região do espectro de emissão da luz (fótons), onde se relaciona a energia emitida em um determinado comprimento de onda. Cada elemento terá um espectro de emissão atômica característico, sendo a concentração proporcional à intensidade desses fótons.

\subsection{Sensibilidade dos Métodos Espectrométricos}

Os métodos de espectrometria são utilizados para determinações qualitativas e quantitativas de mais de 70 elementos químicos. Geralmente possuem detecção na ordem de ppm, ppb e, em alguns casos, em concentrações muito menores. Estes métodos são, além disso, rápidos, convenientes e geralmente de alta sensitividade. Eles podem ser divididos em dois grupos: espectrometria de ótica atômica e espectrometria de massa atômica. [SKOOG, 2004, p.839]

\subsection{Sistema de Introdução de Amostra}

A conversão da amostra da fase líquida para gasosa é efetuada por um dispositivo denominado atomizador.

Há duas classes de aparelho de atomização: atomizador contínuo e atomizador descontínuo. Com atomizadores contínuos, para fontes tais como plasma e chama, as amostras são introduzidas em forma contínua, por exemplo, através de bomba. Com atomizador descontínuo, as amostras são introduzidas de forma descontínua com um aparelho tal como uma seringa ou um autoamostrador. [SKOOG, 2004, p.844].

\subsection{Fonte de Plasma}

Por definição, um plasma é uma mistura de gases condutores contendo uma concentração significativa de cátions e elétrons. No plasma de argônio usado pela espectrometria atômica, íons de argônio e elétrons são as espécies condutoras principais, embora cátions da amostra também contribuam. Íons de argônio uma vez formados no plasma são capazes de absorver força suficiente de uma fonte externa para manter a temperatura em um nível no qual o plasma sustenta maior ionização. No interior do plasma são encontradas temperaturas tão altas como 10.000K. [SKOOG, 2004, p.845].

\section{Plasma acoplado indutivamente}

A figura 3 é um desenho esquemático de uma fonte de plasma acoplado indutivamente. Consiste de três tubos de quartzo concêntricos através do qual passa o fluxo de argônio de uma variação de 11 a $17 \mathrm{l} / \mathrm{min}$. No ambiente do tubo está uma bobina de indução alimentada por um gerador de radiofreqüência de $2 \mathrm{~kW}$, capaz de produzir ondas com frequiências entre 27 a $40 \mathrm{MHz}$. A ionização do fluxo de argônio é inicializada por uma centelha de uma bobina Tesla. Os íons resultantes e os elétrons associados então interagem com o campo magnético (indicação $\mathrm{H}$ na figura 3 ) produzido pela bobina de indução I. Essa interação faz com que os íons e elétrons dentro da bobina fluam nos caminhos fechados anulares representados na figura. 
A temperatura do ICP é alta, mas pode ser termicamente isolada pelo tubo de quartzo. Isto ocorre devido ao fluxo de argônio tangencial ao redor da parede do tubo, como indicado pelas setas na figura 3. O fluxo tangencial resfria as paredes internas do tubo central e os centros radiais do plasma. [SKOOG, 2004, p.845]

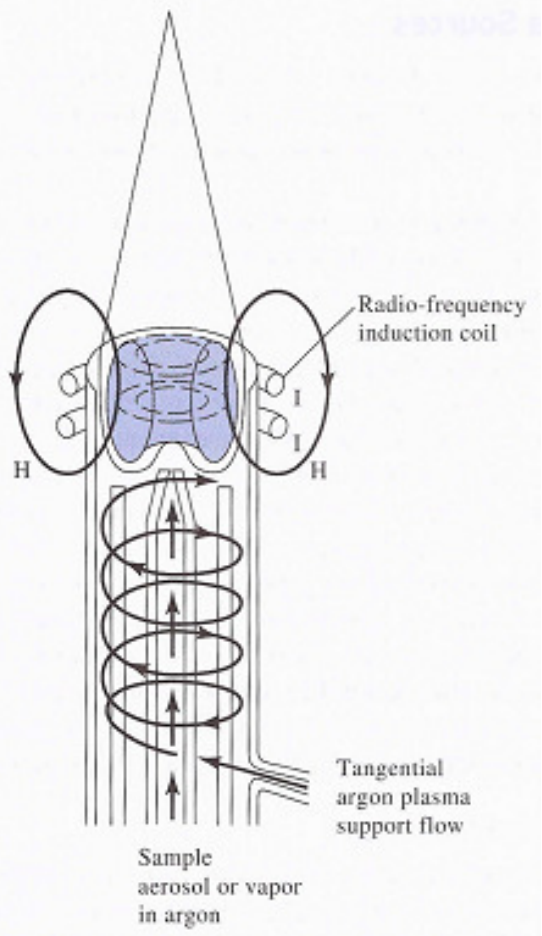

Figura 3: Representação do plasma

[SKOOG, 2004, p.846]

As amostras podem ser introduzidas para dentro do ICP com um fluxo de argônio através do tubo de quartzo central. A amostra pode estar em aerosol, em vapor gerado termicamente ou em pó fino. O mais comum meio de introdução de amostra é o nebulizador de vidro concêntrico. A amostra é transportada pelo efeito Bernoulli. Este processo de transporte é chamado aspiração. O gás a alta velocidade pulveriza o líquido em gotas finas de vários tamanhos, que são então carregadas para dentro do plasma através de arraste. [SKOOG, 2004, p.847].

Existem poucas interferências químicas com fonte de plasma quando comparamos com fonte de centelha. Este fato é devido a: alta velocidade dos íons ou átomos, ao ponto de observação do espectro, a alta temperatura atingida e a completa dessolvatação e vaporização da amostra.

\subsection{Fonte de Centelha}

A excitação da amostra ocorre por eletrodos quando se usa a centelha como fonte. A passagem de eletricidade do eletrodo pela amostra promove a energia necessária para atomizar à amostra e excitar os átomos resultantes para um estado eletrônico mais alto. [SKOOG, 1985, p.306]. 
A análise quantitativa por centelha demanda um controle preciso das muitas variáveis envolvidas na preparação e excitação da amostra. Soma-se a isso o fato de as medidas quantitativas requererem um conjunto de padrões cuidadosamente preparados para a calibração. Esses padrões devem aproximar-se o mais possível da composição e das propriedades físicas das amostras a serem analisadas. [UNIVERSIDADE FEDERAL DO PARÁ].

Em todas as técnicas de análise de espectrometria de emissão a concentração da amostra é determinada com auxílio de uma curva de calibração construída com padrões. A intensidade dos fótons é proporcional à concentração do elemento que gerou esses fótons. Uma vez que padrões e amostras são excitados em condições idênticas pode-se determinar a concentração dos elementos por comparação com a curva de calibração.

A figura 4 apresenta um esquema de um espectrômetro com fonte de centelha aplicado em indústria metalúrgica.

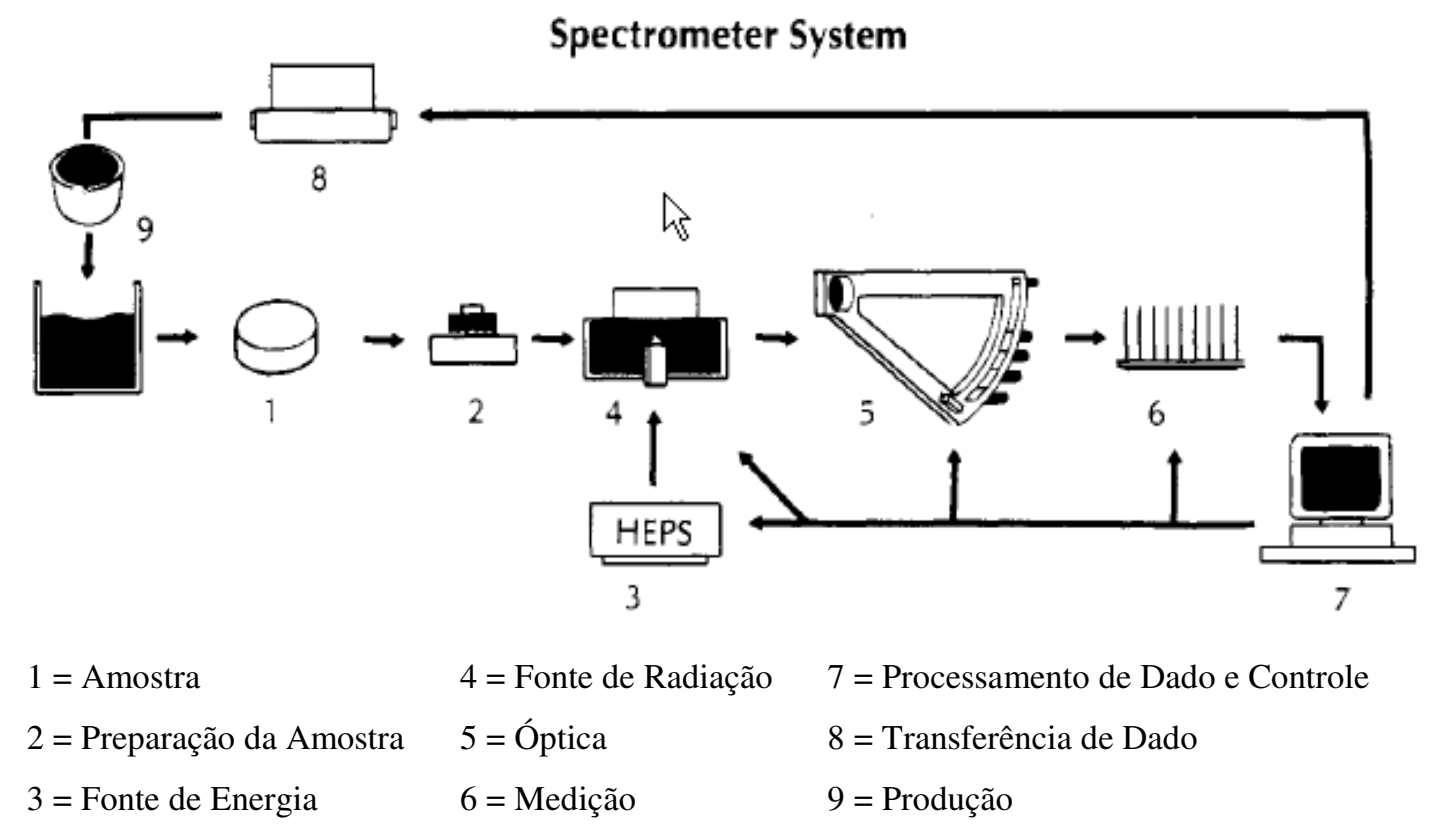

Figura 4: Representação Esquemática de um Spark-AES

[SLICKERS, 1993] 


\section{Parte Experimental}

\subsection{Equipamentos}

Espectrômetro de Emissão Atômica Acoplado com Plasma Induzido do fabricante Spectro, modelo GENESIS

Espectrômetro de Emissão Atômica com Fonte de Centelha do fabricante ThermoARL, modelo ARL3460

Forno de microondas do fabricante CEM, Modelo MARS 5, com recipiente de dissolução tipo HP-500 Plus

\subsection{Condições de Análise}

Emissão Atômica com Plasma: 1350W de potência do Plasma, 12 1/min. de vazão do coolant, 1 1/min. de vazão auxiliar, $0,90 \mathrm{l} / \mathrm{min}$. de vazão do nebulizador, $0,5 \mathrm{l} / \mathrm{min}$. de purga ótica e 3 integrações. O nebulizador utilizado foi o tipo Cross Flow, câmara e tocha resistentes a ácido fluorídrico.

Emissão Atômica com Fonte de Centelha: 2s de fluxo de Argônio, 7s de préintegração, $5 \mathrm{~s}$ de Integração, $0 \mathrm{~s}$ antes da integração, condição da fonte para a préintegração: $3 \mu \mathrm{F}, 0,5 \mathrm{ohm}, 20 \mu \mathrm{H}$ e $450 \mathrm{~V}$, condição da fonte para a integração: $2 \mu \mathrm{F}$, $8 \mathrm{ohm}, 20 \mu \mathrm{H}$ e $400 \mathrm{~V}$, lâmpada de fadiga com intensidade média, tensão dos fototubos com $1000 \mathrm{~V}$.

Para a dissolução por microondas utilizou-se a programação apresentada na tabela 2, com 3 min. de resfriamento programado.

Tabela 2: Programa de dissolução do Forno de Microondas

\begin{tabular}{|c|c|c|c|c|}
\hline Aquecimento (min.) & $\begin{array}{c}\text { Pressão } \\
\text { (PSI) }\end{array}$ & $\begin{array}{c}\text { Dissolução } \\
\text { (min.) }\end{array}$ & $\begin{array}{c}\text { Potência } \\
(\%)\end{array}$ & $\begin{array}{c}\text { Potência } \\
(\mathrm{W})\end{array}$ \\
\hline 10 & 60 & 3 & 80 & 1200 \\
\hline 10 & 90 & 3 & 80 & 1200 \\
\hline 10 & 120 & 3 & 80 & 1200 \\
\hline
\end{tabular}

\subsection{Reagentes Químicos e Materiais}

Ácido Clorídrico PA;

Ácido Nítrico PA;

Ácido Fluorídrico PA;

Água Destilada;

Água Deionizada;

Balão Volumétrico de Teflon de 100mL;

Padrão de aço BCS 401/1;

Proveta de Teflon de 50mL;

Proveta de Teflon de 100mL; 
Frasco de Polietileno de 200mL;

Papel de Filtro Rápido R41;

Funil de Teflon;

Balança Analítica, com precisão de 0,0001g;

Cronômetro;

Capela com Exaustão Vidy;

Gás Argônio;

Lixadeira de bancada;

Lixa 3M grau 60 241D.

\subsection{Preparação das Amostras}

Cada etapa da preparação e análise foi cronometrada individualmente para avaliação do tempo gasto.

\subsubsection{Preparação para Análise no Espectrômetro de Plasma (ICP-OES)}

A preparação partiu da amostra padrão na forma de limalha e envolveu os processos relacionados na tabela 3 com seus respectivos tempos de duração. O tempo programado de resfriamento não é suficiente, os recipientes de pressão ainda apresentam alta pressão e temperatura. Foi preciso um período de resfriamento em capela com exaustão para alcance da temperatura ambiente. Considerou-se como etapas extras a limpeza de balão volumétrico e de frasco de polietileno.

Tabela 3: Etapas de Preparação e Análise

\begin{tabular}{|c|l|}
\hline Etapa & Processo \\
\hline 1 & Lavagem dos recipientes de dissolução \\
\hline 2 & $\begin{array}{l}\text { Pesagem de } 1 \mathrm{~g} \text { de limalha de amostra padrão e adição no recipiente } \\
\text { de dissolução }\end{array}$ \\
\hline 3 & $\begin{array}{l}\text { Preparado de mistura ácida } \\
\text { (água desionizada, HCL, HNO } \text { e HF, } 2: 2: 2: 1)\end{array}$ \\
\hline 4 & $\begin{array}{l}\text { Adição de } 35 \mathrm{ml} \text { da mistura ácida ao recipiente de dissolução, } \\
\text { fechamento e posicionamento no suporte-carrossel }\end{array}$ \\
\hline 5 & $\begin{array}{l}\text { Colocação do suporte-carrossel no interior do forno e conexão do } \\
\text { sensor de pressão }\end{array}$ \\
\hline 6 & Dissolução \\
\hline 7 & Resfriamento em capela com exaustão \\
\hline 8 & $\begin{array}{l}\text { Abertura dos recipientes e transferência da solução ácida para balões } \\
\text { volumétricos de teflon de 100 ml. }\end{array}$ \\
\hline 9 & Filtração com papel de filtro rápido \\
\hline 10 & Medição, 3 medições por amostra \\
\hline Extra 1 & Limpeza de balão volumétrico \\
\hline Extra 2 & Limpeza de frasco de polietileno \\
\hline
\end{tabular}


4.4.2 Preparação de Amostra para Análise no Espectrômetro com Fonte de Centelha A preparação partiu-se da amostra com geometria que possibilitasse seu lixamento, a mesma foi lixada em lixadeira de bancada, obtendo superfície plana e com aparência uniforme.

\section{Resultados}

\subsection{Preparação e Análise no ICP}

Os tempos gastos em média por amostra nas etapas estão apresentados na tabela 4. 
Tabela 4: Tempo Gasto nas Etapas de Preparação

\begin{tabular}{|c|l|}
\hline Etapa & Tempo Gasto \\
\hline 1 & $1 \mathrm{~min} .33 \mathrm{~s}$ \\
\hline 2 & $1 \mathrm{~min} .32 \mathrm{~s}$ \\
\hline 3 & $3 \mathrm{~min} .54 \mathrm{~s}$ \\
\hline 4 & $0 \mathrm{~min} .50 \mathrm{~s}$ \\
\hline 5 & $1 \mathrm{~min} .34 \mathrm{~s}$ \\
\hline 6 & $27 \mathrm{~min} .30 \mathrm{~s}$ \\
\hline 7 & $1 \mathrm{~h}$ \\
\hline 8 & $2 \mathrm{~min} .16 \mathrm{~s}$ \\
\hline 9 & 2 min.27s \\
\hline 10 & 2 min.25s \\
\hline Extra 1 & 1 min.20s \\
\hline Extra 2 & 0 min.44s \\
\hline Total & $1 \mathrm{~h} 46 \mathrm{~min} .05 \mathrm{~s}$ \\
\hline
\end{tabular}

O forno de microondas tem capacidade para 14 recipientes de dissolução, logo o tempo gasto será único com a colocação do carrossel em seu interior, conexão do sensor e dissolução, independente do número de amostras. Nas demais etapas o tempo gasto será proporcional à quantidade da amostra, exceto para preparação da mistura ácida cuja diferença não é significante (depende só da quantidade de cada componente).

Foi estimado em 4 h. 36 min. 36s o tempo para preparação e análise de 14 amostras. Realizando a limpeza dos balões volumétricos e dos frascos de polietileno durante o tempo ocioso da dissolução e resfriamento, o tempo pode ser reduzido para 1h. 44 min. 01s. Para 14 amostras pode-se estimar esse tempo em $4 \mathrm{~h} .07 \mathrm{~min}$. 40s.

\subsection{Preparação e Análise no Espectrômetro de Fonte de Centelha}

Gastou-se 0 min.11s com o lixamento e 1 min. 41 s com a medição por amostra. Sendo assim o tempo estimado para 14 amostras é 26 min.08s.

\subsection{Comparativo de Tempo de Análise}

A diferença de tempo de análise entre os métodos é grande. É possível obter o resultado do teor dos elementos em uma única amostra em aproximadamente 2 minutos pelo Espectrômetro com fonte de Centelha (Spark-AES). Para analisar os mesmos elementos no Espectrômetro com Fonte de Plasma (ICP) pode-se levar em torno de 1 hora e 45 minutos. Mesmo para a capacidade máxima do forno de microondas não há redução significativa desta diferença. Para as 14 amostras pelo Espectrômetro com Fonte de Centelha é possível obter os resultados próximos aos 26 minutos e em torno de 4 horas para o Espectrômetro com Fonte de Plasma. O gráfico 1 apresenta o tempo das análises para uma única amostra entre os instrumentos e o gráfico 2 para a estimativa de 14 amostras, onde se nota a diferença ainda com mais clareza.

É evidente que a grande diferença de tempo para analisar amostra de aço entre os métodos está na preparação, já que os tempos de medição são relativamente próximos, 1 min.52s (fonte de Centelha) contra 2 min.25s (fonte de Plasma). 
Gráfico 1: Comparativo de Tempo de Análise para 1 amostra

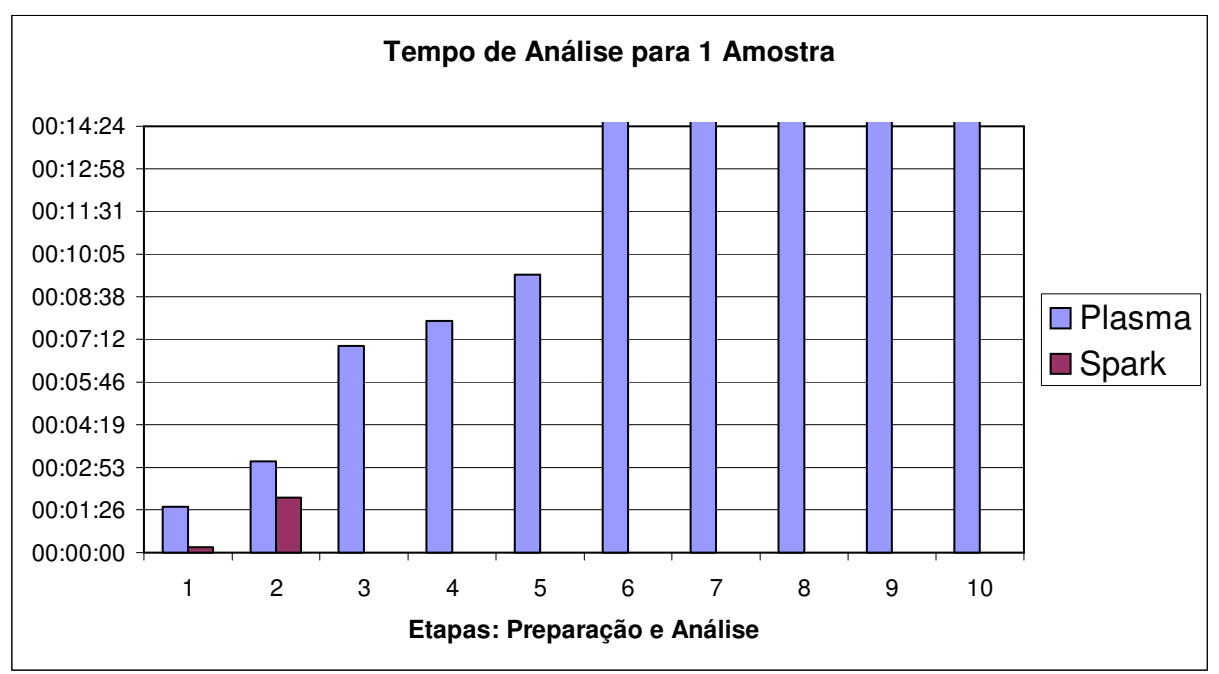

Gráfico 2: Comparativo de Tempo de Análise para 14 amostras

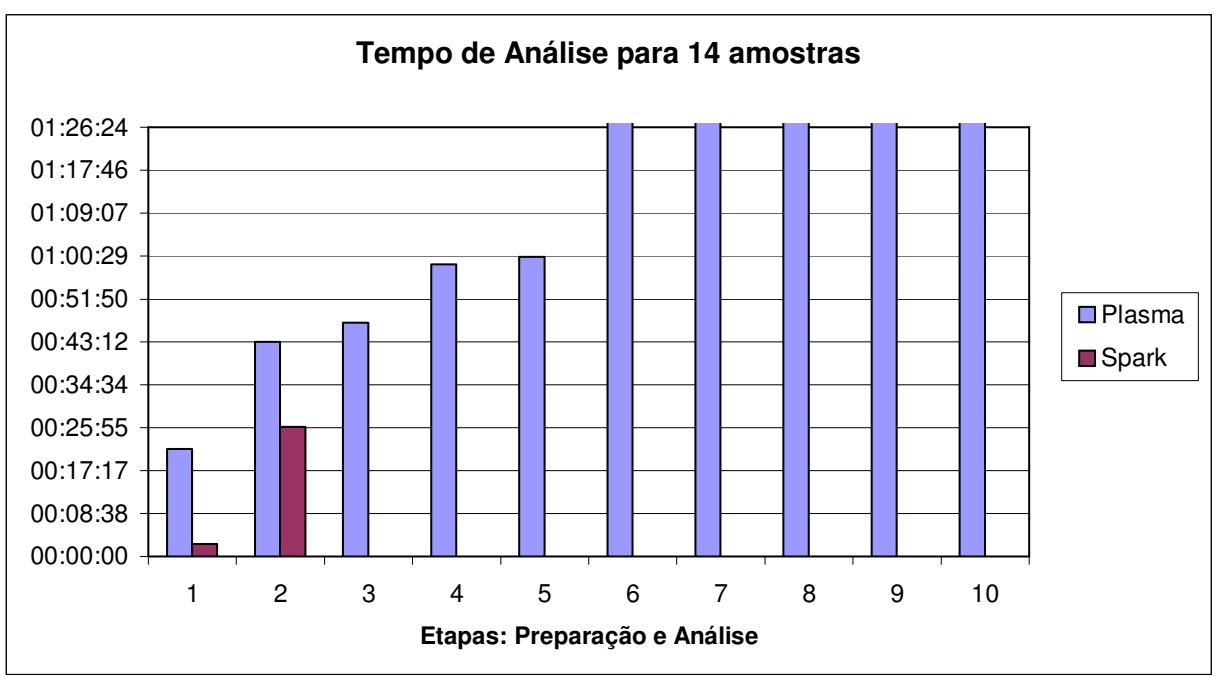

\subsection{Exatidão e Precisão dos métodos}

A tabela 5 mostra os resultados obtidos das concentrações dos elementos estudados e a tabela 6 os valores de recuperação. $O$ valor de recuperação é definido pela razão entre a concentração determinada e a concentração de referência do padrão utilizado.

Tabela 5: Teores Encontrados para a Amostra Padrão BCS401/1

$\begin{array}{lc}\text { Método } & \text { Plasma } \\ \text { Amostra } & 401 / 1 \\ \mathrm{Cr}(\%) & 0,056 \pm 0,001(3) \\ \mathrm{Cu}(\%) & 0,085 \pm 0,003(3) \\ \mathrm{Mn}(\%) & 0,893 \pm 0,014(3) \\ \mathrm{Mo} \mathrm{( \% )} & 0,472 \pm 0,007(3) \\ \mathrm{Ni}(\%) & 0,028 \pm 0,000(3) \\ \mathrm{P}(\%) & 0,046 \pm 0,001(3) \\ \mathrm{Si} \mathrm{( \% )} & 0,409 \pm 0,004(3) \\ \mathrm{V}(\%) & 0,520 \pm 0,009(3)\end{array}$

Centelha
$401 / 1$
$0,056 \pm 0,001(3)$
$0,086 \pm 0,002(3)$
$0,850 \pm 0,009(3)$
$0,464 \pm 0,009(3)$
$0,027 \pm 0,001(3)$
$0,041 \pm 0,001(3)$
$0,402 \pm 0,008(3)$
$0,502 \pm 0,012(3)$

Plasma vs Centelha Diferença $0,000 \%$

$1,163 \%$

$-5,059 \%$

$-1,724 \%$

$-3,704 \%$

$-12,195 \%$

$-1,741 \%$

$-3,586 \%$ 


\section{Tabela 6: Valores de Recuperação}

\begin{tabular}{lrrr} 
Método & Plasma & \multicolumn{1}{c}{ Centelha } & Plasma vs Centelha \\
Amostra & $401 / 1$ & $401 / 1$ & Diferença \\
$\mathrm{Cr}$ & $102,348 \%$ & $102,439 \%$ & $0,089 \%$ \\
$\mathrm{Cu}$ & $96,208 \%$ & $97,358 \%$ & $1,182 \%$ \\
$\mathrm{Mn}$ & $104,728 \%$ & $99,665 \%$ & $-5,080 \%$ \\
$\mathrm{Mo}$ & $100,351 \%$ & $98,652 \%$ & $-1,722 \%$ \\
$\mathrm{Ni}$ & $92,595 \%$ & $\mathbf{8 6 , 4 8 6 \%}$ & $-7,062 \%$ \\
$\mathrm{P}$ & $102,253 \%$ & $93,333 \%$ & $-9,556 \%$ \\
$\mathrm{Si}$ & $99,118 \%$ & $97,451 \%$ & $-1,711 \%$ \\
$\mathrm{~V}$ & $99,770 \%$ & $96,294 \%$ & $-3,610 \%$
\end{tabular}

As diferenças entre as concentrações dos elementos nas duas técnicas estudadas foram menores que $10 \%$, tanto para os teores encontrados quanto para os recuperados (tabelas 3 e 4), exceto para o teor de fósforo (P), que a diferença foi de 12,195\%. Os resultados máximos de recuperação estiveram na faixa de 86.5 a $104.7 \%$.

\subsubsection{Teste de Exatidão empregando o t-Teste}

Um modo de comparar médias é considerar que não existe diferença significativa entre os resultados dos valores médios obtidos.

Neste caso temos duas médias devido a utilização das duas técnicas empregadas: $\mathrm{X}_{1}$, média das determinações com fonte de plasma e $\mathrm{X}_{2}$, média das determinações com fonte de centelha.

Precisamos então testar se a diferença entre $X_{1}$ e $X_{2}$ difere significantemente de zero. Se as duas amostras possuem desvios padrões que não são significativamente diferentes, um teste pode ser efetuado baseado nos valores individuais dos desvios padrões $s_{1}$ e $s_{2}$ :

$$
S^{2}=\left\{\left(n_{1}-1\right) s_{1}^{2}+\left(n_{2}^{2}-1\right) s_{2}^{2}\right\} /\left(n_{1}+n_{2}-2\right)
$$

Pode-se obter um valor de " $\mathrm{t}$ " dado por:

$$
\mathrm{t}=\left(\mathrm{X}_{1}-\mathrm{X}_{2}\right) / \mathrm{s}\left(1 / \mathrm{n}_{1}+1 / \mathrm{n}_{2}\right)^{1 / 2}
$$

onde " $\mathrm{t}$ " tem $\mathrm{n}_{1}+\mathrm{n}_{2}-2$ graus de liberdade

O valor obtido por t na equação acima é comparado com um valor tabelado considerando os graus de liberdade do sistema. $\mathrm{O}$ valor de $\mathrm{t}$ crítico foi retirado da tabela 7 numa distribuição bi-caudal, uma vez que não há expectativa de uma técnica ser mais precisa que a outra. Os valores de $\mathrm{n}_{1}$ e $\mathrm{n}_{2}$ são os mesmos: 3 . Os graus de liberdade são 4 $(3+3-2)$.

Tabela 7: Valores de $t$

\begin{tabular}{|c|c|c|}
\hline $\begin{array}{c}\text { Número de graus } \\
\text { de Liberdade }\end{array}$ & $\mathbf{9 0} \% \mathbf{( 0 , 1 )}$ & $\mathbf{9 5 \%}(\mathbf{0 , 0 5})$ \\
\hline $\mathbf{1}$ & 6,31 & 12,71 \\
\hline $\mathbf{2}$ & 2,92 & 4,30 \\
\hline $\mathbf{3}$ & 2,35 & 3,18 \\
\hline $\mathbf{4}$ & 2,13 & 2,78 \\
\hline $\mathbf{5}$ & 2,02 & 2,57 \\
\hline
\end{tabular}

[MILLER, 1993] 
Considerando os valores da tabela 5 , os valores obtidos de $\mathrm{t}$ para cada elemento são dados na Tabela 8.

Tabela 8: Valores de $t$

\begin{tabular}{|l|r|r|}
\hline Elemento & \multicolumn{1}{|c|}{ t TESTE } & F CRÍTICO \\
\hline $\mathrm{Cr}$ & 0 & 2.13 \\
\hline $\mathrm{Cu}$ & 0.480384 & 2.13 \\
\hline $\mathrm{Mn}$ & 4.47496 & 2.13 \\
\hline $\mathrm{Mo}$ & 1.21529 & 2.13 \\
\hline $\mathrm{Ni}$ & 1.22474 & 2.13 \\
\hline $\mathrm{P}$ & 6.12372 & 2.13 \\
\hline $\mathrm{Si}$ & 1.35554 & 2.13 \\
\hline $\mathrm{V}$ & -3.11769 & 2.13 \\
\hline
\end{tabular}

Pelos resultados mostrados na tabela 8 podemos observar que existem diferenças significativas das médias dos resultados entre as duas técnicas para os elementos Mn, $\mathrm{P}$ e $\mathrm{V}$, uma vez que os valores calculados de $\mathrm{t}$ são maiores que os valores críticos para esses elementos.

\subsubsection{Teste de Precisão}

O teste é efetuado para verificar se existe diferença significativa em relação à precisão entre as duas técnicas estudadas para todos os elementos analisados.

O F-teste considera a razão de duas variâncias, isto é, a razão entre os quadrados dos desvios padrões. A quantidade calculada F é dada por:

$$
\mathrm{F}=\mathrm{s}_{1}{ }^{2} / \mathrm{s}_{2}{ }^{2}
$$

A razão $\mathrm{s}_{1}{ }^{2} / \mathrm{s}_{2}{ }^{2}$ é sempre maior que 1 . A hipótese nula adotada é que as populações das duas amostras são consideradas estatisticamente normais e que as variâncias são iguais. Se a hipótese nula é verdadeira, então as razões entre as variâncias devem ser próximo de 1. Diferenças da razão 1 ocorrem devido a erros aleatórios das técnicas, porém diferenças elevadas não podem ser atribuídas a erros aleatórios. Se o valor calculado de $\mathrm{F}$ excede a um certo valor crítico (tabelado) então a hipótese nula é rejeitada. $\mathrm{O}$ valor crítico de $\mathrm{F}$ depende do tamanho de ambas amostras, do nível de significância e do tipo de teste efetuado.

Os valores para $\mathrm{P}=0.05$ são mostrados pela tabela 9 . Considerando que não há expectativa de uma ou outra técnica ser mais precisa, os valores do valor crítico com $\mathrm{P}=0.05$ foram retirados considerando o teste bi-caudal. $\mathrm{O}$ valor crítico de $\mathrm{F}$ é determinado usando os graus de liberdade do numerador e do denominador, para ambos será o número de duplicatas menos $1(3-1=2)$, na razão $\mathrm{F}=\mathrm{s}_{1}{ }^{2} / \mathrm{s}_{2}{ }^{2}$. Usando esses graus de liberdade e a tabela 9 encontra-se o valor de F crítico igual a 39.

Tabela 9: Valores críticos de $F(P=0,05)$

\begin{tabular}{|c|c|c|c|c|c|}
\hline & $\boldsymbol{\vartheta}_{\mathbf{1}}$ & $\mathbf{1}$ & $\mathbf{2}$ & $\mathbf{3}$ & $\mathbf{4}$ \\
\hline $\boldsymbol{\vartheta}_{\mathbf{2}}$ & $\mathbf{1}$ & 647,8 & 799,5 & 864,2 & 899,6 \\
\hline & $\mathbf{2}$ & 38,51 & 39,0 & 39,17 & 39,25 \\
\hline & $\mathbf{3}$ & 17,44 & 16,04 & 15,44 & 15,10 \\
\hline & $\mathbf{4}$ & 12,22 & 10,65 & 9,979 & 9,364 \\
\hline
\end{tabular}

[MILLER, 1993] 
A tabela 10 mostra os valores determinados de $\mathrm{F}$ para cada elemento e o F crítico.

\begin{tabular}{lcc}
\multicolumn{3}{c}{ Tabela 10: Resultados do teste F } \\
Elemento & VALORES DE F & F CRÍTICO \\
$\mathbf{C r}$ & 1.00 & 39 \\
$\mathbf{C u}$ & 2.25 & 39 \\
$\mathbf{M n}$ & 2.42 & 39 \\
$\mathbf{M o}$ & 0.60 & 39 \\
$\mathbf{~ N i}$ & 1.00 & 39 \\
$\mathbf{P}$ & 1.00 & 39 \\
$\mathbf{S i}$ & 0.25 & 39 \\
$\mathbf{V}$ & 0.56 & 39
\end{tabular}

Pelos resultados pode-se observar que para todos os elementos os valores calculados de $\mathrm{F}$ são menores que o valor crítico, mostrando assim que não há diferença significativa entre os métodos em relação à precisão.

\section{Conclusões}

Pode-se dizer que as precisões das duas técnicas são semelhantes. Os resultados das médias das concentrações dos elementos entre as duas técnicas mostraram diferenças significativas para os elementos $\mathrm{Mn}, \mathrm{P}$ e $\mathrm{V}$. Essa exatidão pode estar relacionada a qualquer dispersão dos elementos na parte metálica da amostra, quando se utilizou a centelha como fonte de excitação.

Houve grandes diferenças no tempo de análise, no preparo de amostra, tipo e forma de amostra e aplicação.

A Espectrometria de Emissão Atômica com Fonte de Centelha é uma técnica específica, uma vez que analisa apenas um tipo de material (metálico), e na forma sólida. A aplicação da técnica é simples, rápida e de baixo custo, uma vez que os materiais utilizados são mínimos, o que é uma grande vantagem para aplicação em controle de produção de metais, onde se analisa apenas um tipo de material. Além disso como em todo processo de produção requerem-se menor tempo e baixo custo.

A Espectrometria de Emissão Atômica com Fonte de Plasma Acoplado Indutivamente não apresenta restrições quanto à forma e tipo de amostra, o que vai influenciar é a etapa de preparação. A vantagem mais notável é a aplicação diversificada, uma vez que se pode analisar qualquer material que seja possível convertê-lo para forma líquida.

Apesar de suas particularidades, as técnicas possuem um grande potencial analítico e uma ampla aplicação. 


\section{Referências Bibliográficas}

SKOOG, D. A. Fundamentals of Analytical Chemistry. $8^{a}$ Edição. Canadá. Editora Thomson. 2004. 1179 p.

SKOOG, D. A. Principles of Instrumental Analysis. $3^{\text {a }}$ Edição. Estados Unidos da América. Editora Saunders College Publishing. 1985. 548 p.

JEFFERY, G. H. Bassett, J. Mendham, J. Denney, R. C. Vogel - Análise Química Quantitativa. 5 Edição. Rio de Janeiro - RJ. Livros Técnicos e Científicos Editora S.A. 1992.

PETRY, C. F. Determinação de Elementos Traços em Amostras Ambientais por ICP OES. Disponível em: http://www.lume.ufrgs.br/handle/10183/4604. Acessado em: 24 de set. de 2009.

TREVIZAN, L. C. Matias, T. B. Nóbrega, J. A. Análise de Aço Inoxidável por Espectrometria de Emissão Óptica com Plasma Acoplado Indutivamente com Configuração Axial. Águas de Lindóia - SP. 30ª Reunião Anual - Sociedade Brasileira de Química. 31/05 a 03/06/2007. Disponível em: http://sec.sbq.org.br/cdrom/30ra/resumos/T0944-1.pdf. Acessado em 10 de out. de 2009.

VANCE, E. R. Emission Spectroscopy in the Steel Industry. Ohio - EUA. The Ohio Journal of Science. Volume 52. $\mathrm{N}^{\mathrm{o}}$ 3. p.114 - 117. Maio de 1952. Disponível em: https://kb.osu.edu/dspace/bitstream/1811/3922/1/V52N03_114.pdf. Acessado em 13 de out. de 2009.

DOUCET, F. R. Belliveau, T. F. Fortier, J. Hubert, J. Comparative Study of Laser Induced Plasma Spectroscopy and Spark-Optical Emission Spectroscopy for Quantitative Analysis of Aluminium Alloys. Journal of Analytical Atomic Spectrometry. 2004. 19. p.499 - 501. 02/03/04. Disponível em: http://www.rsc.org/publishing/journals/JA/article.asp?doi=b315619b. Acessado em 21 de maio de 2009.

Faculdade Federal do Pará. Espectrometria de Emissão Atômica. Disponível em: http://www.ufpa.br/ccen/quimica/espectroscopia\%20de\%20emissao.htm. Acessado em 02 de maio de 2009.

MILLER, J.C. Miller, J.N. Statistics for Analytical Chemistry, Edit: Ellis Horwwod PTR Prentice Hall. 1993. 233p.

MELLOR, J.W. Química Inorgânica Moderna. $3^{\text {a }}$ Edição. Rio de Janeiro. Editora Globo. 1955. $1061 \mathrm{p}$.

SILVA, A. L. C. Mei, P. R. Aços e Ligas Especiais. 2ª Edição. Sumaré - SP. Editora Eletrometal S.A. Metais Especiais. 1988.

SLICKERS, K.A. Spectrochemical Analysis in the Metallurgical Industry. Pure \& Appl. Chem. Vol. 65. N¹2. p.2443-2452, 1993. Printed in Great Britain. 